\title{
Delegated Portfolio Management with Socially Responsible Investment Constraints *
}

\author{
A. Fabretti, S. Herzel \\ SEFeMeQ, \\ University of Rome "Tor Vergata". \\ Via Columbia 2 - 00133 Roma, ITALY \\ e-mail:annalisa.fabretti@uniroma2.it \\ e-mail:stefano.herzel@uniroma2.it
}

\begin{abstract}
We consider the problem of how to establish compensation for a portfolio manager who is required to restrict the investment set, for example, because of socially responsible screening. This is a problem of Delegated Portfolio Management where the reduction of investment opportunities to the subset of sustainable assets involves a loss in expected earnings for the portfolio manager, compensated by the investor through an extra bonus on the realized return. Under simple assumptions on the investor, manager and market, we compute the optimal bonus as a function of the managers risk aversion and expertise, and of the impact of portfolio restriction on the mean-variance efficient frontier.
\end{abstract}

JEL classification: G11 - Portfolio Choice; Investment Decisions.

D86 - Economics of Contract: Theory

*Acknowledwgement: This study has been funded by the Foundation for Strategic Environmental Research, MISTRA. 


\section{Introduction}

Socially Responsible Investment (SRI), that is the selection of assets of firms satisfying some socially responsible criteria, is receiving increasing attention from private and institutional investors. While mainstream economic theory considers Social Responsibility as a non-financial issue that should most likely lead to extra costs, several studies argue that it may have a positive financial value. Mill (2006) investigated this point by examining the financial performance of a socially responsible investment over time. Platinga and Scholtens (2001), Stone et al. (2001) and Bauer et al. (2007) compared the performances of SRI funds to those of conventional funds. Kempf and Osthoff (2007) tried to separate portfolio performance from fund manager ability and compared purposely designed SRI and non-SRI portfolios. The general picture is still unclear and the evidence is mixed because of different data sets and different interpretations. However, it is a fact that not only researchers, but also a substantial number of investors, believe in the possibility of "doing well while doing good". On the other hand, portfolio managers appear reluctant to include SRI in their investment strategies, as reported by RImetrics (2008). It is evident that any restriction in the investment universe set by the investor for whatever reason makes the job of portfolio managers more difficult and may also reduce their remuneration if it depends on the performance of investment strategies. Portfolio managers play a key role in implementing SRI, and hence it is important to study how to provide suitable incentives for designing policies geared to fostering SRI.

Let us assume that an investor wishes to invest her wealth according to a socially responsible criterion. To this end, she will probably hire a portfolio manager because he has better knowledge of the market, and will pay him a proportion of the return produced by his investment decisions. As the investment opportunities are restricted by the investors requests, the manager would rather accept a mandate from another, non-green, investor, unless he is paid an extra bonus. How is this bonus related to the characteristics of the market, to the risk propensity of the agents, and to the skills of the managers? These are the kind of questions that we address in the present paper. Ours is a stylized version of a rather common situation where an institutional investor wishes to maximize the expected return while complying with some non-financial constraints. The investor may also be interpreted as a government establishing a fiscal bonus to encourage some investments more than others. For an empirical analysis on managers attitudes toward policy regulations, we refer the reader to Sundblad et al. (2009).

To establish the model, we followed the lines proposed by the classical Delegated Portfolio Management (DPM) literature; see Stracca (2005) for a review. The typical problem of DPM is that of designing an appropriate contract when the portfolio managers information and efforts are not directly observable by the investor. As a result, an appropriate contract should motivate the manager into making a costly effort to gather information and to use this information in choosing a portfolio with desirable risk characteristics (for the investor). The literature mainly focuses on optimal contract functions and their effects, such as in Stoughton (1993), Wei and Litiwari (2009), and Admati and Pfleiderer (1997), but it has, rather surprisingly, devoted little attention to the restriction commonly found in investment policies that defines contracts between investors and managers, as shown by Almazan et al (2004). One notable exception is Gomez and Sharma (2006), who studied the implications of establishing short-selling constraints.

Our study aimed to formulate a model that would be simple enough to obtain ex- 
plicit results, but also sufficiently structured to address some important issues, such as the impact of the managers risk aversion and skill as well as of the loss in diversification opportunities, on the compensation scheme when a constrained investment mandate is proposed. To this end, we considered a single-period model where the allocation universe consists of risky assets: some "green", according to given SRI criteria, and others "nongreen ". Of course, restricting the investment set reduces the expected utility for both investor and manager, and generates a "sustainability cost". While the green-conscious investor is willing to bear this cost, the manager is driven by purely financial motivations and will ask for some compensation that we call a "green bonus". Adopting a linear sharing rule, assuming a risk-neutral investor, a risk-averse portfolio manager, and normally distributed returns, we obtained an explicit formula for the "green bonus". The bonus is given by the sum of two factors, the first one related to lost opportunities in terms of investment and risk diversification, while the second related to the missed exploitation of the managers knowledge of the market. We showed how to measure the first component in the case of the screening of financial assets from S\&P500 of December 2006 according to some SRI criteria. Analyzing the second component, we noted that managers with greater expertise in green assets should require a lower bonus than equally skilled, but less "green-focused", managers. That is to say, as observed by Kreps (1997), stronger intrinsic motivations demand smaller extrinsic incentives. When considering more contractual features, such as short-selling constraints, or a different contract, such as one with limited liability, we are still able to solve the problem, but not with an explicit solution. In the last part of the present paper we analyze some of the consequences due to the introduction of some extra features, observing, for example, that a limited liability contract may be better than a linear one, not only for the manager, but also for the investor.

\section{The model}

We considered a one-period economy, with two agents: a risk neutral investor, and a portfolio manager with an exponential utility function $u(x)=-\exp (-\alpha x)$, where the coefficient of risk aversion coefficient $\alpha$ is common knowledge. There are $n$ risky assets with return $\mathbf{X}$ distributed as a multivariate normal random variable with mean $\overline{\mathbf{X}}$ and variance $\Sigma$ and a riskless asset with return $R$. The risky investment set can be partitioned into two disjoint subsets, the first consisting of $p$ "green" assets with return $\mathbf{P}$, normally distributed with mean $\overline{\mathbf{P}}$ and variance $\Sigma_{P}$ and by $q$ "non-green "assets $\mathbf{Q}$, normally distributed with mean $\overline{\mathbf{Q}}$ and variance $\Sigma_{Q}$. That is, we have

$$
\mathbf{X}=\left(\begin{array}{c}
\mathbf{P} \\
\mathbf{Q}
\end{array}\right), \quad \overline{\mathbf{X}}=\left(\begin{array}{c}
\overline{\mathbf{P}} \\
\overline{\mathbf{Q}}
\end{array}\right), \quad \Sigma=\left(\begin{array}{cc}
\Sigma_{P} & \Sigma_{P Q} \\
\Sigma_{P Q}^{T} & \Sigma_{Q}
\end{array}\right)
$$

where $\Sigma_{P Q}$ is an $p \times q$ matrix and the superscript $T$ represents transposition. Our setting is an extension of Bhattachary and Pfleiderer (1985) to the case of $n$ risky assets and the distinction between "green" and "non-green" assets.

The manager receives a noisy signal on the return $\mathbf{X}$, given by

$$
\mathbf{S}=\mathbf{X}+\epsilon
$$

where $\mathbf{X}$ and $\epsilon$ are uncorrelated and $\epsilon$ is a normal $n$-dimensional random variable with mean zero and variance $\Sigma_{\epsilon}$. The random vector $\epsilon$ is the noise of the signal, hence the skill 
of a manager is represented by the variance $\Sigma_{\epsilon}$. The manager decides on the investment only after observing a value $S$.

The conditional distribution of $\mathbf{X}$ given $\mathbf{S}=S$ is a normal random variable with mean

$$
\mathbf{M}(S)=\overline{\mathbf{X}}+\Sigma \Sigma_{S}^{-1}(S-\overline{\mathbf{X}})
$$

where $\Sigma_{S}=\Sigma+\Sigma_{\epsilon}$ and variance

$$
V=\Sigma-\Sigma \Sigma_{S}^{-1} \Sigma
$$

Note that $V$ is a symmetric positive definite matrix that does not depend on $S$.

To distinguish between green and non-green asset components we define

$$
\mathbf{M}(S)=\left(\begin{array}{c}
\mathbf{M}_{\mathbf{P}}(S) \\
\mathbf{M}_{\mathbf{Q}}(S)
\end{array}\right), \quad V=\left(\begin{array}{cc}
V_{P} & V_{P Q} \\
V_{P Q}^{T} & V_{Q}
\end{array}\right) .
$$

The SRI investor allocates a capital sum $W_{0}$ to the manager with a mandate to restrict trading to green and risk-free assets. The manager's compensation is

$$
f(W):=A R+b W
$$

where the parameter $A$ is a fixed amount received at the beginning of the period and $b$ is the fee on the realized wealth, that is given by

$$
W\left(\omega_{P}\right)=\omega_{P}^{T} \mathbf{P}+\left[W_{0}-\mathbf{1}_{p}^{T} \omega_{P}\right] R
$$

where $\omega_{P}$ is a $p$ dimensional vector representing the allocation in green assets and $\mathbf{1}_{p}$ is the $p$ dimensional vector with all components equal to one. We assume that the manager can also choose a second contract without investment restrictions, whose payoff is

$$
r\left(W^{\prime}\right):=A R+b_{0} W^{\prime}
$$

and where

$$
W^{\prime}(\omega)=\omega^{T} \mathbf{X}+\left[W_{0}-\mathbf{1}_{n}^{T} \omega\right] R,
$$

where $\omega$ is an $n$ dimensional vector representing the allocation in the whole investment set. We define "green bonus "as the quantity

$$
\Delta:=b-b_{0}
$$

that measures the compensation required by the manager for the restriction on the investment.

The manager observes the signal only after accepting the contract. After this, he determines the optimal allocation $\omega_{P}^{*}$ by optimizing his expected utility

$$
\mathbb{E}\left[u\left(f\left(W\left(\omega_{P}\right)\right)\right) \mid \mathbf{S}=S\right]
$$

allocating the wealth $W_{0}$ in green assets. The decision on whether or not to accept the contract must be taken before observing the signal on the basis of the expected utility over all possible signals. Of course, the decision depends on the bonus $\Delta$ offered by the investor. Hence the manager accepts the green contract if the participation constraint

$$
\mathbb{E}[u(f(W))] \geq \mathbb{E}\left[u\left(r\left(W^{\prime}\right)\right)\right]
$$


is satisfied. We refer to the right hand side of (6) as the manager's "reservation utility".

Note that both $W$ and $W^{\prime}$ depend on the manager's allocation $\omega_{P}$ and $\omega$, respectively, which in turn depend on the observed signal $S$ and also on the matrix $\Sigma_{\epsilon}$. Moreover the optimal allocation $\omega_{P}^{*}$ (resp. $\omega^{*}$ ) is a function of the contract parameter $b$ (resp. $b_{0}$ ), thus $\omega_{P}^{*}$ is a function of $\Delta$. In the following, we explicitly indicate only one or two of these dependencies at a time for the sake of a simpler notation.

The principal maximizes the expectation of her final wealth after rewarding the manager. We remark that we do not impose any short-selling constraint and that the manager's compensation may also turn out to be negative: that is, the contract does not have limited liability. We will relax these hypotheses later. Putting all the pieces together, we can state the problem of the investor

$$
\begin{gathered}
\max _{\Delta} \mathbb{E}\left[W\left(\omega_{P}^{*}(\Delta, \mathbf{S})\right)-f\left(W\left(\omega_{P}^{*}(\Delta, \mathbf{S})\right)\right)\right] \\
\omega_{P}^{*}(\Delta, S)=\arg \max _{\omega_{P}} \mathbb{E}\left[u\left(A R+\left(b_{0}+\Delta\right) W\left(\omega_{P}\right)\right) \mid \mathbf{S}=S\right] \\
\mathbb{E}[u(f(W))] \geq \mathbb{E}\left[u\left(r\left(W^{\prime}\right)\right)\right]
\end{gathered}
$$

\section{The Optimal Bonus}

In this section we solve problem (7),(8) and (9) in two steps: first by assuming that the manager does not receive any private signal and then by also considering private information. By proceeding in two steps we will see that the green bonus can be broken down into two factors. The first factor takes into account differences in investment opportunities, while the second depends on the signal being unexploited because of the investment restriction. That is, we can distinguish which part of the bonus depends on the properties of market features and which one on the manager's expertise.

\subsection{The case of managers without private information}

Here we assume that the investor and the manager have access to the same information on returns on investment and that the investor needs to hire the manager because she cannot trade directly. This situation is equivalent to the case where the signal is so noisy that the observation of $\mathbf{S}$ does not provide any valuable information to the manager.

The investor's problem in this case is given by

$$
\begin{gathered}
\max _{\Delta} \mathbb{E}\left[W\left(\omega_{P}^{*}(\Delta)\right)-f\left(W\left(\omega_{P}^{*}(\Delta)\right)\right)\right] \\
\omega_{P}^{*}(\Delta)=\arg \max _{\omega_{P}} \mathbb{E}\left[u\left(A R+\left(b_{0}+\Delta\right) W\left(\omega_{P}\right)\right)\right] \\
\mathbb{E}[u(f(W))] \geq \mathbb{E}\left[u\left(r\left(W^{\prime}\right)\right)\right]
\end{gathered}
$$

the solution of which is given by the following result.

Proposition 1 The solution to the problem (10) (11) (12) is

$$
\bar{\Delta}_{0}=\frac{1}{2 \alpha W_{0} R} \Psi
$$


where

$$
\begin{aligned}
\Psi & =\left(\mathcal{S}-\mathcal{S}_{P}\right), \\
\mathcal{S}_{P}=\left(\overline{\mathbf{P}}-R \mathbf{1}_{p}\right)^{T} \Sigma_{P}^{-1}\left(\overline{\mathbf{P}}-R \mathbf{1}_{p}\right) \text { and } \mathcal{S} & =\left(\overline{\mathbf{X}}-R \mathbf{1}_{n}\right)^{T} \Sigma^{-1}\left(\overline{\mathbf{X}}-R \mathbf{1}_{n}\right) .
\end{aligned}
$$

Proof. First we prove that the manager's participation constraint (12) is satisfied for $\Delta \geq \bar{\Delta}_{0}$, then we show that the principal's expected utility is decreasing with respect to $\Delta$.

The expected utility on the left hand side of (12) is given by

$$
\mathbb{E}\left[-e^{-\alpha f(W)}\right]=-e^{-\alpha A R} \cdot e^{-\alpha b \mu_{W}+\frac{(\alpha b)^{2}}{2} \sigma_{W}^{2}}
$$

since $W$ is normally distributed.

The solution of (11) is

$$
\omega_{P}^{*}=\frac{1}{\alpha\left(b_{0}+\Delta\right)}\left(\overline{\mathbf{P}}-R \mathbf{1}_{p}\right) \Sigma_{P}^{-1} .
$$

Hence, the maximal expected utility, obtained by substituting $\omega_{P}^{*}$ into (15), is

$$
\mathbb{U}_{0}(f(W)):=\mathbb{E}\left[-e^{-\alpha f\left(W\left(\omega_{P}^{*}(\Delta)\right)\right)}\right]=-e^{-\alpha A R} \cdot e^{-\frac{\mathcal{S}_{P}}{2}} \cdot e^{-\alpha\left(b_{0}+\Delta\right) W_{0} R} .
$$

Similarly, the reservation utility on the right hand side of (12), is given by

$$
\mathbb{U}_{0}\left(r\left(W^{\prime}\right)\right):=\mathbb{E}\left[-e^{-\alpha r\left(W^{\prime}\right)}\right]=-e^{-\alpha A R} \cdot e^{-\frac{\mathcal{S}}{2}} \cdot e^{-\alpha b_{0} W_{0} R} .
$$

The expected value of the principal's wealth, obtained by substituting the optimal solution $\omega_{P}^{*}$ into $(10)$, is

$$
E\left[W_{P}\right]=\left(1-\left(b_{0}+\Delta\right)\right)\left[W_{0} R+\frac{\mathcal{S}_{P}}{\alpha\left(b_{0}+\Delta\right)}\right]-A R
$$

that is a decreasing function of $\Delta$, hence the principal chooses the smallest $\Delta$ satisfying constraint (12). Hence, substituting (16) and (17) into (12) we get (13).

The value $\bar{\Delta}_{0}$ is the minimal bonus necessary to convince an agent (who does not receive any private signal) to accept a "green" mandate. Note that $\mathcal{S}$ and $\mathcal{S}_{P}$ are the highest possible Sharpe Ratios (squared) obtained on the whole market and on the "green" market, respectively. Hence, $\bar{\Delta}_{0}$ is always positive. The Sharpe Ratio difference measures the loss of expected risk adjusted returns. Figure 1 gives an interpretation of the effect of the green bonus. The left-hand panel shows the green efficient frontier (dashed line) which is of course below the conventional one (solid line). The two optimal allocations (green and conventional) belong to two different utility curves. In the right-hand panel it can be seen how the green bonus $\bar{\Delta}_{0}$ shifts the green efficient frontier upwards (dash-dot line) so that the new green optimal allocation now belongs to the same utility curve as the conventional one. 
The optimal green bonus $\bar{\Delta}_{0}$ is inversely proportional to risk aversion and to $W_{0}$ that represents the Asset Under Management. Setting $W_{0}=1$ in (13) we get the green bonus as a percentage of the total return on investment - a more interesting quantity to examine. The inverse relation with risk aversion is explained by the fact that more risk averse managers tend to invest less in risky assets and more in risk-free assets, hence they are less worried about the loss in diversification and, consequently, demand a smaller extra bonus.

Let us now compute the green bonus as a function of the manager's risk aversion by estimating the quantities appearing in (13) on data from the US market. Here we adopt the methodology proposed by Herzel et al. (2010), to which we refer for further details. We consider monthly returns of the assets belonging to the S\&P 500 Index from 1993 to 2006 and we estimate their variance matrix $\Sigma_{t}$ through the widely used Fama-French (1992) factorial model. To estimate the expected returns of the assets we make the assumption that the S\&P 500 Index is efficient. This assumption avoids the pitfalls related to the estimates of expected returns in factor models, pointed out, for example by Kandel and Stambaugh (1995): it is in fact an assumption of market equilibrium that is also adopted as a starting point of the popular Black-Litterman model (1992) for portfolio selection. Hence, the expected rate of returns of the assets are given by

$$
\mu_{t}=R_{t}+\alpha_{M} w_{t}^{m k t}
$$

where $R_{t}$ is the risk-free rate, $\alpha_{M}$ is the "market risk-aversion ", that is, the risk-aversion of a representative investor that puts all his wealth in the Index and $w_{t}^{m k t}$ represents the relative market capitalizations. Next, we consider the scores provided by KLD Research and Analytics, a rating agency specialized in sustainability issues for firms traded in the US market. To compare the effects of different screening policies, we restricted the investment set, originally composed of 488 assets, according to three criteria, corresponding to the three classic dimensions of social responsibility, Environment, Social, Governance. More specifically, by excluding all the assets that raised any concern, we discharged a total of 147 in the case of Environmental Screening, 336 for Governance, and 417 for Social Screening. Finally, we solved the mean-variance optimization problems to obtain the tangent portfolios on each of the three efficient frontiers and compute the corresponding Sharpe ratios $\mathcal{S}_{P}$. Figure 2 represents the amount of the green bonus $\bar{\Delta}_{0}$ for December 2006 as a function of relative risk aversion $\alpha / \alpha_{M}$. The case considered shows that the green bonus should be rather small (never higher than 20 basis points), because the loss in terms of Sharpe Ratio due to the screening is, for all the cases considered, small despite the fact that the number of assets discharged is generally high. This last observation holds even more generally, see Herzel et al.(2010) for a statistical study on this issue. These numerical results depend on the rating system adopted by one particular rating agency. The criteria used for formulating a rating index are of the utmost importance for any investor. We do not discuss this issue here as it goes beyond the scope of our work, but we refer to Bhagat at al. (2008) for a deep analysis of the various problems connected to the choice of Corporate Governance index.

[FIGURE 2 ABOUT HERE] 


\subsection{The case of private information}

Now we consider the case of managers receiving a private signal on the future returns. In this case, the optimal bonus is given by the following

Proposition 2 The solution of the optimisation problem (7), (8) and (9) is

$$
\bar{\Delta}=\frac{1}{2 \alpha W_{0} R}(\Psi+\Phi)
$$

where $\Psi$ is given in (14) and

$$
\Phi=\log \left(\frac{\operatorname{det}\left(V^{-1} \Sigma\right)}{\operatorname{det}\left(V_{P}^{-1} \Sigma_{P}\right)}\right) .
$$

Proof. The optimal allocation after observing the signal $S$ is

$$
\omega_{P}^{*}=\frac{1}{\alpha\left(b_{0}+\Delta\right)} V_{P}^{-1}\left(\mathbf{M}_{P}(S)-R \mathbf{1}_{p}\right),
$$

for the green contract and

$$
\omega^{*}=\frac{1}{\alpha b_{0}} V^{-1}\left(\mathbf{M}(S)-R \mathbf{1}_{n}\right)
$$

for the conventional contract. The corresponding expected utilities are

$$
\mathbb{E}[u(f(W)) \mid \mathbf{S}=S]=-e^{-\alpha A R} \cdot e^{-\alpha\left(b_{0}+\Delta\right) W_{0} R} \cdot e^{-\frac{\mathcal{S}_{P}(S)}{2}}
$$

and

$$
\mathbb{E}\left[u\left(r\left(W^{\prime}\right)\right) \mid \mathbf{S}=S\right]=-e^{-\alpha A R} \cdot e^{-\alpha b_{0} W_{0} R} \cdot e^{-\frac{\mathcal{S}(S)}{2}},
$$

where

$$
\mathcal{S}(S)=\left(\mathbf{M}(S)-R \mathbf{1}_{n}\right)^{T} V^{-1}\left(\mathbf{M}(S)-R \mathbf{1}_{n}\right)
$$

and

$$
\mathcal{S}_{P}(S)=\left(\mathbf{M}_{P}(S)-R \mathbf{1}_{p}\right)^{T} V_{P}^{-1}\left(\mathbf{M}_{P}(S)-R \mathbf{1}_{p}\right) .
$$

To compute the expectations of (20) and (21) we consider the random variables $\mathbf{M}(\mathbf{S}) \sim$ $N(\overline{\mathbf{X}}, Q)$ and $\mathbf{M}_{P}(\mathbf{S}) \sim N\left(\overline{\mathbf{P}}, Q_{P}\right)$, where $Q=\Sigma \Sigma_{S}^{-1} \Sigma$ and $Q_{P}$ is the matrix composed of the first $p$ rows and $p$ columns of $Q$. Let us define

$$
\varphi(A, B, \nu):=\mathbb{E}\left[e^{-\frac{v^{T} A v}{2}}\right]
$$

where $A$ is a $n \times n$ positive definite matrix and $v$ is a $n$-dimensional normal random variable with mean $\nu$ and variance $B$. A standard computation gives

$$
\varphi(A, B, \nu)=\frac{e^{-\frac{c}{2}}}{\sqrt{\operatorname{det}\left(A B+I_{n}\right)}},
$$

where

$$
c=\nu^{T}\left(B^{-1}-B^{-1}\left(A+B^{-1}\right)^{-1} B^{-1}\right) \nu
$$


and $I_{n}$ is the identity matrix of dimension $n$. Since $\mathbf{M}(\mathbf{S})$ and $\mathbf{M}_{P}(\mathbf{S})$ are normally distributed,

$$
\mathbb{E}[\mathbb{E}(u(f(W)) \mid \mathbf{S})]=-e^{-\alpha A R} \cdot e^{-\alpha\left(b_{0}+\Delta\right) W_{0} R} \cdot \varphi\left(V_{P}^{-1}, Q_{P}, \mu_{P}\right)
$$

and

$$
\mathbb{E}\left[\mathbb{E}\left(u\left(r\left(W^{\prime}\right)\right) \mid \mathbf{S}\right)\right]=-e^{-\alpha A R} \cdot e^{-\alpha b_{0} W_{0} R} \cdot \varphi\left(V^{-1}, Q, \mu\right)
$$

where $\mu=\overline{\mathbf{X}}-R, \mu_{P}=\overline{\mathbf{P}}-R$. Hence, the participation constraint (9) is equivalent to

$$
e^{-\alpha \Delta W_{0} R} \cdot \varphi\left(V_{P}^{-1}, Q_{P}, \mu_{P}\right) \leq \varphi\left(V^{-1}, Q, \mu\right)
$$

that is satisfied if - and only if - the bonus $\Delta$ is greater than or equal to

$$
\bar{\Delta}=\frac{1}{\alpha W_{0} R} \log \left(\frac{\varphi\left(V_{P}^{-1}, Q_{P}, \mu_{P}\right)}{\varphi\left(V^{-1}, Q, \mu\right)}\right) .
$$

Now observe that the principal's expected wealth is

$$
E\left[W_{P}\right]=\left(1-\left(b_{0}+\Delta\right)\right)\left[W_{0} R+\frac{E\left[\mathcal{S}_{P}(\mathbf{S})\right]}{\alpha\left(b_{0}+\Delta\right)}\right]-A R
$$

which is a decreasing function of $\Delta$; hence, the optimal green bonus is given by the smallest $\Delta$ satisfying (9), that is $\bar{\Delta}$. By substituting the definitions of $\Psi$ and $\Phi$ into (23) we get (18).

Proposition 2 shows that the optimal green bonus is equal to a constant that depends on the manager's risk aversion $\alpha$, on the return of the risk free asset $R$, and on the asset under management $W_{0}$, multiplied by the sum of two terms. The first term, $\Psi$, is not affected by the manager's skill and relates to the loss in investment opportunity as perceived by the investor. The second term $\Phi$ reflects the cost of unexploited information and only depends on the manager's ability. Since $\mathcal{S}_{P}(S) \leq \mathcal{S}(S)$ for any $S$, we have $E\left[e^{-\mathcal{S}_{P}(\mathbf{S})}\right] \geq E\left[e^{-\mathcal{S}(\mathbf{S})}\right]$ and consequently $\varphi\left(V_{P}^{-1}, Q_{P}, \mu_{P}\right) \geq \varphi\left(V^{-1}, Q, \mu\right)$ and

$$
\frac{\sqrt{\operatorname{det}\left(V^{-1} \Sigma\right)}}{\sqrt{\operatorname{det}\left(V_{P}^{-1} \Sigma_{P}\right)}} \geq \frac{e^{\frac{\mu_{P}^{T} \Sigma_{P}^{-1} \mu_{P}}{2}}}{e^{\frac{\mu^{T} \Sigma^{-1} \mu}{2}}}
$$

for any $\mu$. Considering that the right-hand side is equal to one when $\mu=0$, we find that the left-hand side is always greater than one and it follows that $\Phi \geq 0$.

Finally, we observe that when $\Phi$ is equal to zero, that is the manager is not missing any investment opportunity because of unexploited information, the green bonus is equal to the quantity $\bar{\Delta}_{0}$ given by $(13)$.

\section{Manager's expertise and selection}

In this section we propose a definition of manager's expertise and we discuss the problem of manager's selection. We call "total expertise "of a manager the quantity:

$$
H:=\frac{\operatorname{det}(\Sigma)}{\operatorname{det}(V)}
$$


Note that $H$ is always greater than one. Similarly, we call $H_{P}=\operatorname{det}\left(V_{P}^{-1} \Sigma_{P}\right)$ the "green expertise ". Two managers with different skills (represented by $\Sigma_{\epsilon}$ ) may have the same total expertise $H$, if, for instance, they have a comparable level of knowledge regarding different industrial sectors. The factor $\Phi$ can be written as

$$
\Phi=\log \left(\frac{H}{H_{P}}\right),
$$

that is, it is proportional to the ratio between total and green expertise, and hence it depends on the manager's relative knowledge of the green market.

Note that $\Phi$ increases with the total expertise $H$ and decreases with the green expertise $H_{P}$. This means that if two managers have the same total expertise $H$, the one with higher expertise in green assets requires a lower bonus. In the extreme case of $H_{P}=H$, when the agent's expertise is concentrated in green assets, no efficiency bonus is required.

A simple example, illustrated by Figure 3, shows the opposite influence of the correlation on the two terms making up the green bonus. Consider a model with only two assets, one green and the other non-green, with variances, respectively $\sigma_{P}^{2}$ and $\sigma_{Q}^{2}$. Let us denote the correlation between the two assets by $\rho$ and suppose that the variance of the signal is a diagonal matrix, with elements $\sigma_{\epsilon, P}^{2}$ and $\sigma_{\epsilon, Q}^{2}$. From a straightforward computation we get that

$$
\Phi=\log \left(1+\left(1-\rho^{2}\right) \frac{\sigma_{Q}^{2}}{\sigma_{\epsilon, Q}^{2}}\right) .
$$

We see that the maximum is reached when $\rho$ is equal to zero. The logic behind this is that if there is no correlation, any information on the non-green asset (represented by the ratio $\frac{\sigma_{Q}^{2}}{\sigma_{\epsilon, Q}^{2}}$ ) is useless if the manager can invest only in the green asset; hence, he will require the highest possible compensation. On the other hand, when the correlation is +1 or -1 , any information on the non-green asset is useful also for the green asset; hence, no expertise is wasted, and the agent will not require any extra compensation. Continuing with the example, we observe that in this case the second term of the optimal bonus is

$$
\Psi=\frac{\left(\rho \sqrt{\mathcal{S}_{P}}-\sqrt{\mathcal{S}_{Q}}\right)^{2}}{1-\rho^{2}}
$$

where $\sqrt{\mathcal{S}_{P}}$ and $\sqrt{\mathcal{S}_{Q}}$ represent the Sharpe ratios of, respectively, the green and non-green asset. The term $\Psi$ is unbounded as $\rho$ approaches +1 or -1 . The reason is that, taking opposite positions in the two assets, the manager could set up a strategy with a vanishing variance and a positive return and he would require a very big bonus to miss such a good opportunity. The minimum value is reached for $\rho=\sqrt{\frac{\mathcal{S}_{P}}{\mathcal{S}_{Q}}}$, if $\mathcal{S}_{P}<\mathcal{S}_{Q}$, or otherwise for $\rho=\sqrt{\frac{\mathcal{S}_{Q}}{\mathcal{S}_{P}}}$, that is when the correlation is such that the investment in the non-green asset is of little interest ( when $\mathcal{S}_{P}<\mathcal{S}_{Q}$ and $\rho=\sqrt{\frac{\mathcal{S}_{P}}{\mathcal{S}_{Q}}}$ ) or of no interest at all ( when $\mathcal{S}_{P}>\mathcal{S}_{Q}$ and $\left.\rho=\sqrt{\frac{\mathcal{S}_{Q}}{\mathcal{S}_{P}}}\right)$.

[FIGURE 3 ABOUT HERE] 
The most important problem for any investor is that of selecting a skilful manager. In our setting, the relevant quantities to be determined by the investor are total expertise $H$ and green expertise $H_{P}$. In any practical situation, assessing the ability of a portfolio manager is a very hard task. Kothari and Warner (2001) highlight the difficulties of determining statistically significant differences between managers' performances by comparing respective realized returns. For example, they show that even if a portfolio manager has enough talent to generate greater portfolio performances of 100 basis points per annum, using the best available techniques it is not possible to demonstrate this with a significant degree of statistical confidence.

In our model it is possible for the investor to implement a simple bidding strategy to attract a manager with a sufficient green efficiency $\hat{H}_{P}$ supposing that the remaining quantities affecting the green bonus in Formula (18) are known ${ }^{1}$. Suppose that the investor identified a group of portfolio managers with the same risk aversion $\alpha$ and the same total expertise $H$ and that she wants to hire one of them whose green expertise is at least equal to $\hat{H}_{P}$. Then, by offering a green bonus with a $\Phi_{0}$ computed by setting $H_{P}=\hat{H}_{P}$ in (24), she will attract all managers with a green expertise greater than $\hat{H}_{P}$, because the efficiency term is decreasing with respect to $H_{P}$, as shown in Figure 4 . The main message here is that, from a group of equally skilled managers, if an investor wants to pick those more focused on green investments, she should not offer more than $\Phi_{0}$ to compensate the loss in efficiency. Should the investor offer more than $\Phi_{0}$, managers with less knowledge of the green sector will also accept her offer. This is reminiscent of the arguments of Kreps (1997) on intrinsic motivations and extrinsic incentives: managers with a stronger intrinsic motivation to invest in green assets demand a smaller extrinsic incentive in terms of extra bonus, and a higher extrinsic incentive can sometimes have a negative result, reducing intrinsic motivations.

\section{[FIGURE 4 ABOUT HERE]}

\section{Short-selling constraints and limited liability}

In this section we study the effects of some modifications to the contract between investor and manager on the green bonus, on the investment strategies and on the expected utilities of the agents. We will consider the case of asymmetrical contracts and of short-selling constraints. For the sake of simplicity we assume that the manager does not have any private information on future returns, that is we modify problem (10), (11) and (12). The modified problems can be efficiently solved by a standard numerical optimization algorithm.

\subsection{Limited liability}

It is often the case that portfolio managers' contracts are subject to a limited liability; that is, the managers are not personally liable for any losses deriving from their investment

\footnotetext{
${ }^{1}$ While some of these quantities, such as the variances and covariances of asset returns, can be estimated through standard statistical methods, as we did for the empirical measure of $\Delta_{0}$ in Section 3, others, like the risk aversion $\alpha$ should be determined by analyzing the managers' attitudes toward risk. The estimation of the total efficiency $H$ is probably the harder quantity to estimate. In our setting it may be inferred from the reservation utility declared by the managers.
} 
strategy. To model this we consider the payoffs

$$
f^{a}(W)=A R+\left(b_{0}+\Delta\right) \max (W, 0)
$$

for the green contract, and

$$
r^{a}(W)=A R+b_{0} \max (W, 0)
$$

for the reservation contract. Hence, we modify the original problem by substituting these functions into (10), (11) and (12) and we run several tests to study the differences between this type of contract and the linear one studied above. Figure 5 shows the results obtained for a market with only two risky assets, one green, $P$, one non-green, $Q$, when the parameters are set to $A=0.3, R=1, W_{0}=1, \sigma_{P}=\sigma_{Q}=1, b_{0}=0.2, \rho=0.3, \mu_{Q}=1.2$ and the expected return on the green asset $\mu_{P}$ varies. The top-left panel compares the wealth invested in asset $P$ for the two cases of linear contract and of limited liability as a function of the ratio of expected returns. In both cases the position increases as $\mu_{P}$ increases, starting from a negative exposure, i.e. a short position, and then becoming positive as the green asset becomes more attractive. We remark that the absolute value of the investment is always greater in the case of a limited liability contract; that is, this type of contract encourages the manager to adopt more risky positions. The top-right panel shows the utilities of the investor as a function of the ratio of returns for the two contracts. We note that the utility obtained for limited liability is almost always greater than the one obtained for the linear case. The same result holds for the utility of the manager, as shown in the bottom-left panel. The bottom-right panel displays the green bonuses for the limited and the unlimited liability contract, showing that the limited liability contracts bonus is always greater than that for the linear contract.

Summarizing the results, confirmed by all of our tests, limited liability encourages the manager to take on more risk, is usually better in terms of utility for both the manager and the investor, and requires a higher green bonus than the linear contract.

\section{[FIGURE 5 ABOUT HERE]}

\subsection{Short-selling constraints}

Short-selling is sometimes prohibited by market rules or also restricted by the investment mandate. In particular, for the case we are examining, it is conceivable for a green investor to prevent her portfolio manager from adopting a bearish strategy by short-selling the green assets. One can take into consideration several possibilities for such a constraint. Here we limit our analysis to two cases that we believe are more significant. In the first case no short-selling on both green and non-green assets is imposed only by the green investor. Therefore it is prohibited only for a manager accepting the green contract. In the second case market rules prevent short-selling on any asset, and hence such a constraint also affects the conventional contract.

Figure 6 represents a typical example of the first case (no short-selling only for the green contract) for a market with only two assets and the following set of parameters $A=0.3, R=1.11, W_{0}=1, \sigma_{P}=\sigma_{Q}=1, b_{0}=0.2, \rho=0.3, \mu_{Q}=1.2$, with the expected return of the green asset $\mu_{P}$ varying from $50 \%$ to $120 \%$ of the return of the non-green asset $\mu_{Q}$. The top left panel compares the allocations in the green asset with (dotted line) 
and without (solid line) short-selling. We see that the constraint becomes active when the expected return of the green asset becomes lower than $80 \%$ of the return of the nongreen asset. The bottom-left panel shows that the utility of the principal with short-selling (dotted line) is lower than the corresponding utility without short-selling (solid line) when the short-selling constraint is active. This is due to the fact that the investor must offer a higher bonus to compensate the manager for the missed opportunity of short-selling, as shown in the right-hand panel. The manager's utility is not affected by such a constraint because any loss is compensated by the bonus. We see that the green bonus is always greater than or equal to the one that should be paid when no constraints are imposed.

\section{[FIGURE 6 ABOUT HERE]}

When the short-selling constraint is imposed also on the conventional contract, there are two main differences from the previous case. The first is that the manager's utility may be lower than the corresponding one in the case of no constraints, because the bonus compensates the manager for losses in the reservation utility that, in this case, may also be affected by the constraint. The second one, represented in Figure 7 is that the green bonus may also be smaller than the corresponding one without constraints, for the same reason stated above; in fact, it depends on the difference between the manager's utilities obtained with the green and with the conventional contract. When short-selling constraints are applied, the difference can be both smaller or greater than in the unconstrained case.

\section{[FIGURE 7 ABOUT HERE]}

\section{Conclusion}

This work is a first attempt to formulate a model of Delegated Portfolio Management with constraints on the investment universe. In particular, we focused on investment restrictions motivated by Social Responsibility by studying the problem of determining the "green bonus ", that is, the minimal incentive required by a portfolio manager to renounce to investments in non-green assets. This bonus may also be interpreted as a sustainability cost. In our model, there are two reasons why the portfolio manager may suffer a loss owing to restrictions on his investment set: the first depends on the assets available and can be represented by a reduction in the mean-variance efficient frontier. The second depends on the managers knowledge of the assets, which we represented by a privately observed signal on future returns. We showed that, in our simple setting, both factors contribute to the bonus, together with the managers attitude towards risk. We also proved that managers with better knowledge of the green market will accept a green contract for a smaller bonus than a manager with the same skills but with less experience in the green sector. This last observation led us to make some general considerations on the problem of manager selection. A very important issue is testing our results on real data. We proposed one way to estimate the first factor composing the green bonus for the case of assets making up the S\&P500 index in the month of December 2006 for three criteria of exclusions based on the Social Responsible scores provided by KLD, a rating agency. We observed that, even for very high level screening, the level of the bonus does not have to be higher than 20 basis points. Tests of other implications of our model, relating to managers knowledge, are more difficult to implement and beyond the scope of this paper. When more features, 
such as limited liability or short-selling constraints, are added to the contract, the problem does not have an explicit solution, but we can still solve it numerically. Our tests show that a contract with limited liability encourages the manager to invest more boldly and may thus be better for both manager and investor. More numerical tests, not shown in the paper, suggest that considering a risk-averse investor does not affect the results of the green bonus. The effect of other distributions for the returns, such as with heavy tails, is more difficult to analyze in the present setting and requires further research.

\section{References}

[1] A. Admati and P. Pfleiderer (1997), "Does it all add up? Benchmarks and the Compensation of Active Portfolio Managers", The Journal of Business , 70, 323-350.

[2] A. Almazan and K. C. Brown and M. Carlson and D. A. Chapman (2004), "Why constraint your mutual fund manager", Journal of Financial Economics, 73, 289-321.

[3] S. Bhagat and R. Romano and B. Bolton (2008), "The Promise and Perils of Corporate Governance Indices, Columbia Law Review, 108, 1803-1882.

[4] S. Bhattachary and P. Pfleiderer (1985), "Delegated Portfolio Management", Journal of Economic Theory 36, 1-25.

[5] R. Bauer and J. Derwall and R. Otten (2007), "The Ethical Mutual fund Performance Debate: new evidence from Canada", Journal of Business Ethics, 70, 111-124.

[6] Black F., Litterman R (1992), "Global Portfolio Optimization", Financial Analyst Journal, 48, 28-43

[7] Eurosif (2008), "European SRI Study 2008", www.eurosif.org, [accessed 20 November 2009].

[8] E. F. Fama and K. R. French (1992), "The cross section of expected stock returns", Journal of Finance, 47, 427-465.

[9] J. P. Gomez and T. Sharma (2006), "Portfolio Delegation under Short-selling Constraints", Economic Theory, 28, 173-196.

[10] R. Heinkel and N. Stoughton (1994), "The Dynamincs of Portfolio Management Contracts", The Review of Financial Studies, 7, 351-387.

[11] S. Herzel, M. Nicolosi, C. Stărică (2010), "The cost of sustainability on optimal portfolio choices", SIRP working paper.

[12] S. Kandel and R.F. Stambaugh (1995), "Portfolio Inefficiency and the Cross-Section of Expected Returns", Journal of Finance, 50, 157-184.

[13] A. Kempf and P. Osthoff (2007), "The effect of Socially Responsible Investing on Portfolio Performance", European Financial Management, 13(5), 908-922.

[14] S.P. Kothari and J. B. Warner. (2001) " Evaluating Mutual Fund Performance," Journal of Finance, 56, 1985-2010. 
[15] D. M. Kreps (1997), "Intrinsic motivation and Extrinsic Incentives", The American Economic Review, 87, 359-364.

[16] G. A. Mill (2006), "The Financial Performance of a Socially Responsible Investment over time and a possible link with corporate social responsibility", Journal of Business Ethics, 63, 131-148.

[17] A. Platinga and B. Scholtens (2001), " Socially Responsible Investment and Management style of Mutual funds in the Euronext stock market", http://som.eldoc.ub.rug.nl/FILES/reports/themeE/2001/01E17/01E17.pdf, [accessed 12 March 2010].

[18] RImetrics (2008), "Responsible Investment Report, An analysis and measurement of competencies and practices of leading asset managers", http://www.rimetrics.com/[accessed 20 November 2009].

[19] S . A. Ross (1973), "The Economic Theory of Agency: The Principal's Problem", American Economic Review, 63, 134-39.

[20] B. K. Stone and J. B. Guerard and M. N. Gultekin and G. Adams (2001), " Socially Responsible Investment Screening: Strong Empirical Evidence of no significant cost for Actively managed value-focused portfolio", http://www.institutionalshareowner.com/presentations/Socially-ResponsibleInvestment-actively-managed-value.pdf, [accessed 10 March 2010]

[21] N. M. Stoughton (1993), "Moral Hazard and the Portfolio Management Problem", The Journal of Finance, 48, 2009-2028.

[22] L. Stracca (2005) "Delegated Portfolio Management: A Survey of the Theoretical Literature", EBC Working Paper, 520, available at SSRN: http://ssrn.com/abstract $=781088$.

[23] E.L. Sundblad and T. Garling and A. Biel and M. Hedesstrom (2009), "Portfolio managers' attitudes towards policy regulations of environmental reporting", Sustainable Investment and Corporate Governance Working Papers , 3, 1-15.

[24] C. Wei Li and A. Tiwari (2009), "Incentive Contracts in Delegated Portfolio Management", Review of Financial Studies, forthcoming, available at SSRN: http://ssrn.com/abstract $=972312$

[25] R. B. Wilson (1968) "The theory of syndicates", Econometrica, 36, 119-132. 

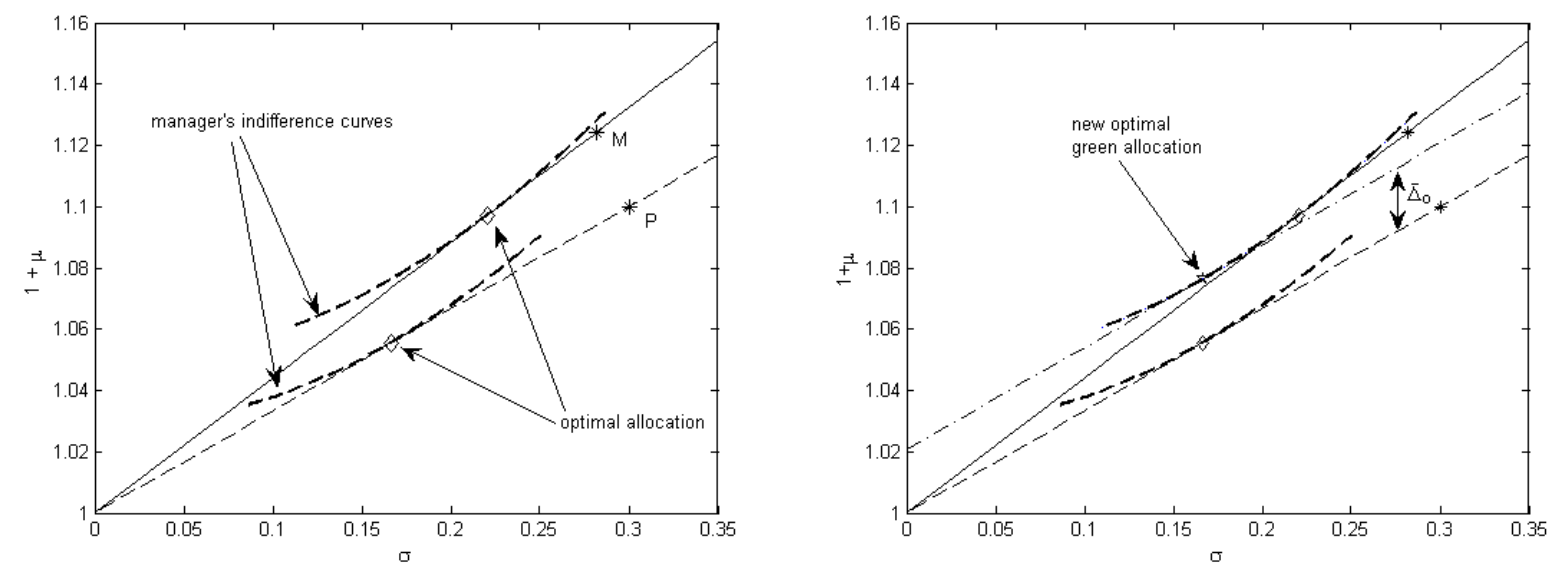

Figure 1: Effects of the green bonus on the manager's allocation and utility. The solid and dashed lines are, respectively, the conventional and green efficient frontiers. The left panel shows the conventional optimal allocation and the green one when no bonus is offered. Note that the utility provided by the green allocation is lower than the conventional one. The right-hand panel shows that the minimum green bonus $\bar{\Delta}_{0}$ shifts the green efficient frontier upwards (dash-dotted line) so that the green optimal allocation provides the same level of utility as the optimal conventional one. 


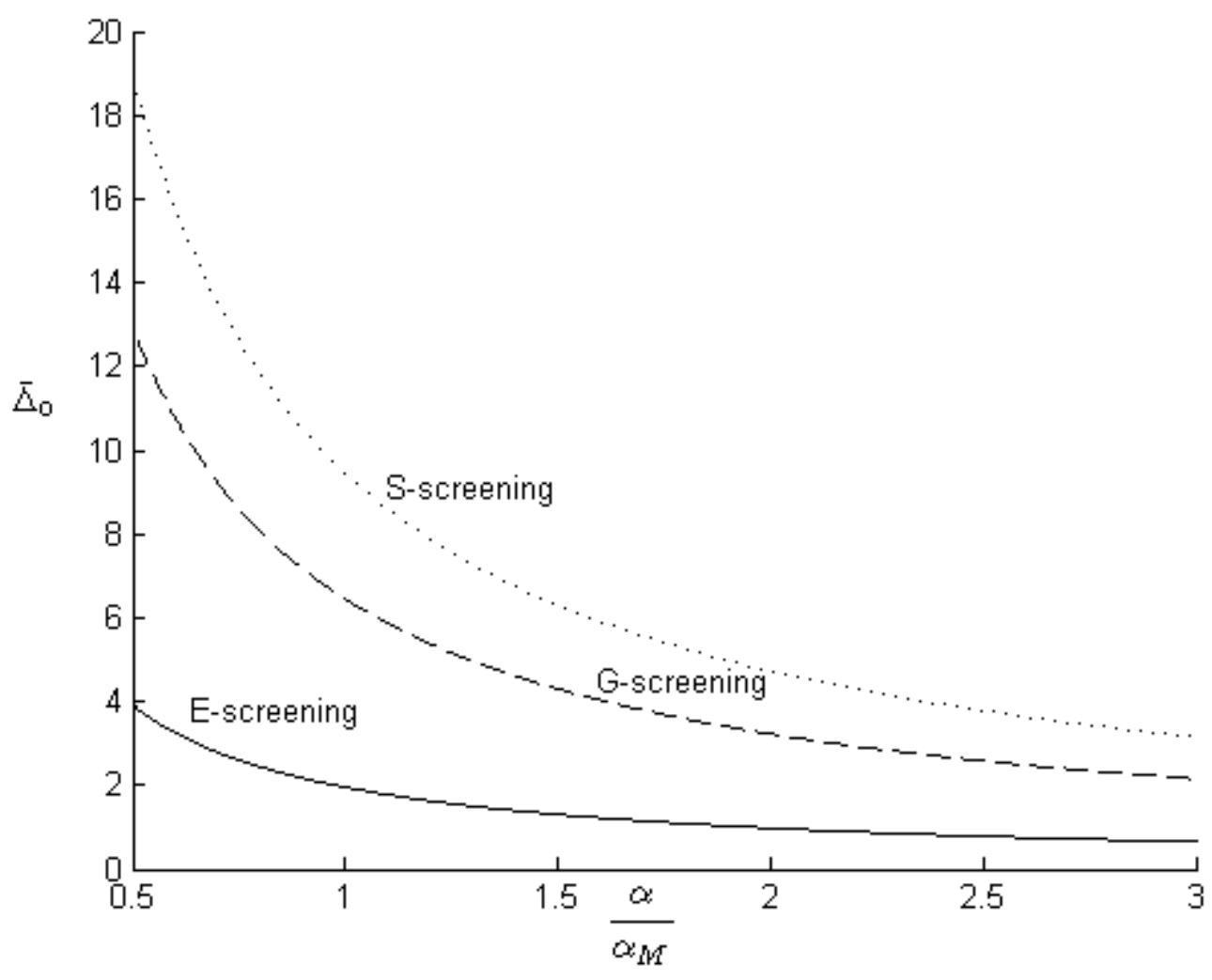

Figure 2: The minimum green bonus $\bar{\Delta}_{0}$ (in basis points) as a function of $\alpha / \alpha_{M}$, where $\alpha$ is the manager's risk aversion and $\alpha_{M}$ is the market risk aversion, for three different screenings. The screening process has excluded the $30 \%, 69 \%, 85 \%$ of assets respectively for the Environmental, the Governance and the Social dimensions. Data are from S\&P500 assets December 2006 screened by using KLD scores. 


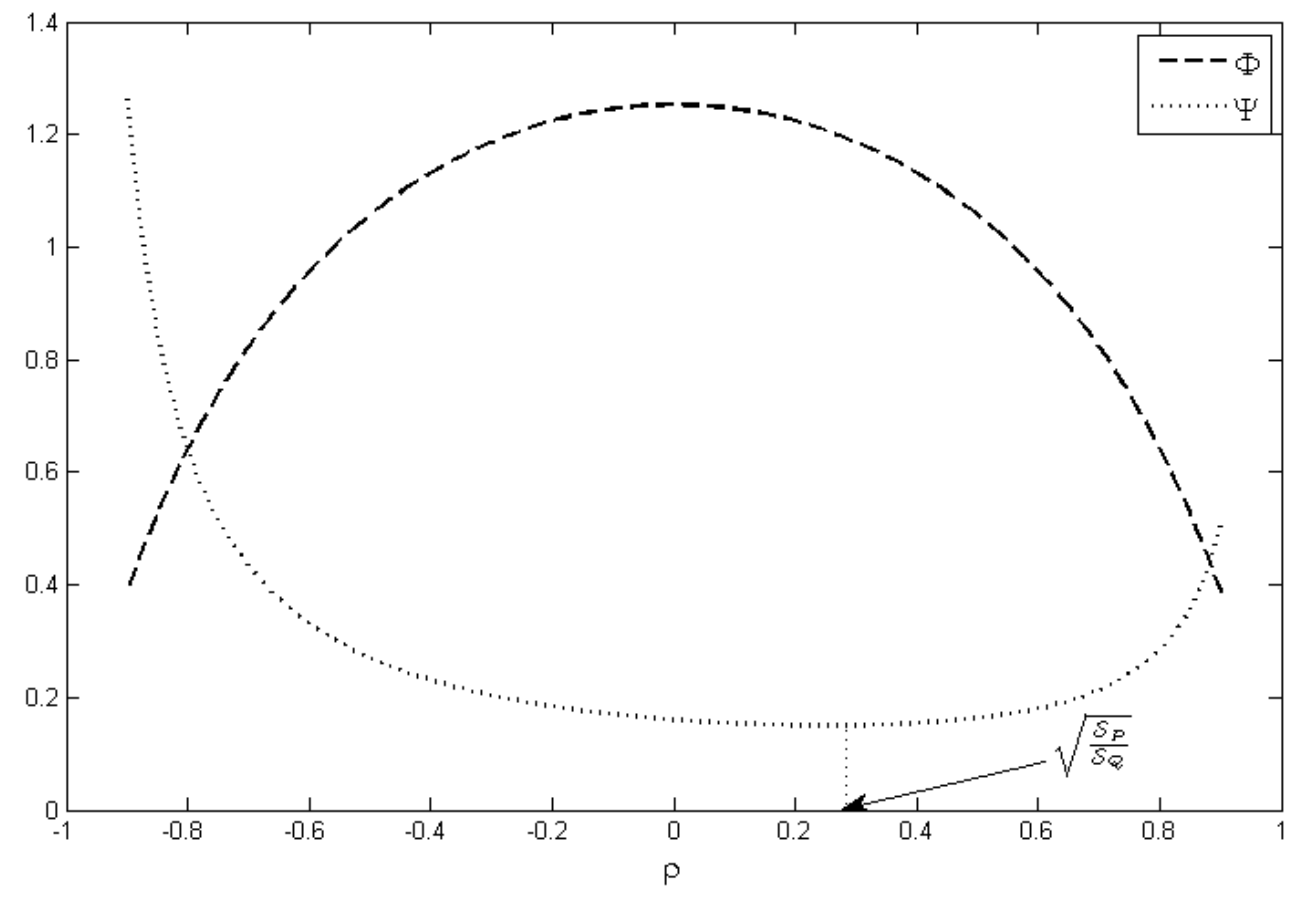

Figure 3: The effect of the correlation on the green bonus. The picture represents the terms $\Psi$ (dotted line) and $\Phi$ (dashed line) as a function of the correlation $\rho$, in the case of a market with only one green asset with Sharpe Ratio $\sqrt{\mathcal{S}_{P}}$ and one non-green asset with Sharpe Ratio $\sqrt{\mathcal{S}_{Q}}$, with $\mathcal{S}_{P}<\mathcal{S}_{Q}$. Parameters are $\mu_{P}=1.1, \mu_{Q}=1.4, \sigma_{P}^{2}=\sigma_{Q}^{2}=1$, $\sigma_{\epsilon, P}^{2}=0.3, \sigma_{\epsilon, Q}^{2}=0.4$ 


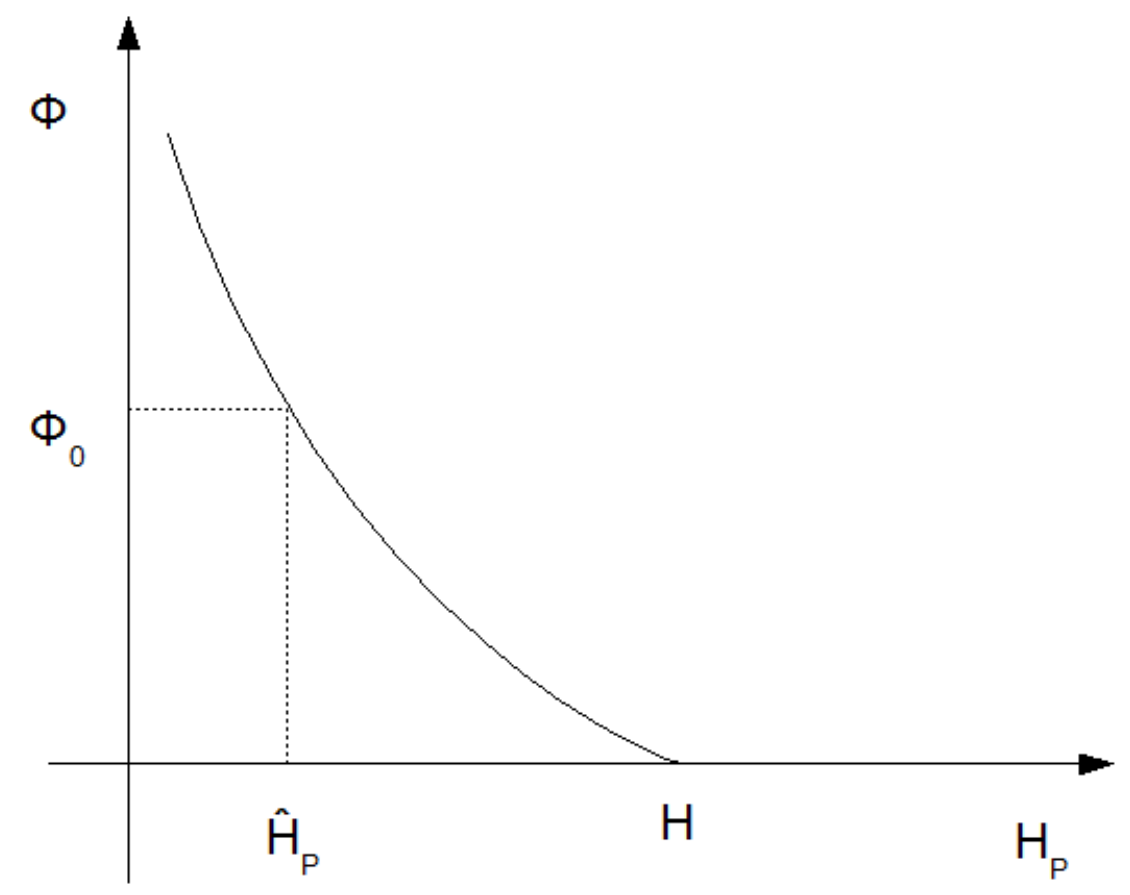

Figure 4: The term $\Phi$ for managers with total efficiency $H$ as a function of their green expertise $H_{P}$. By setting $\Phi=\Phi_{0}$ the investor is sure to attract managers with a green efficiency that is greater or equal to $\hat{H}_{P}$. 

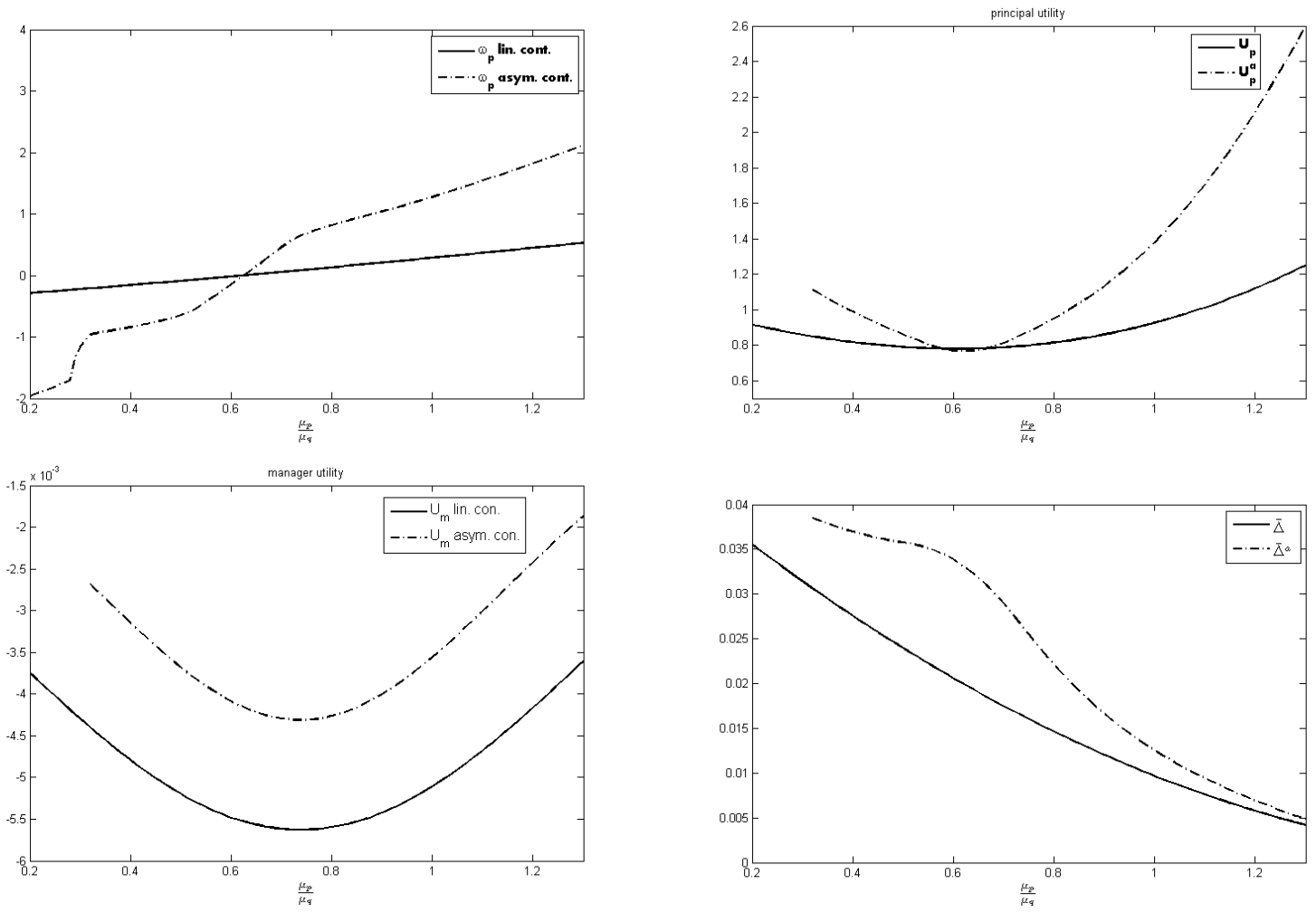

Figure 5: The allocation (top-left panel), the principal's utility (top-right), the manager's utility (left-bottom) and the optimal $\Delta$ are plotted against the ratio between $\mu_{P}$ and $\mu_{Q}$ when the contract is linear (solid line) and when the contract has limited liability (dashed line). Limited liability encourages the manager to take on more risk and provides a greater utility for both manager and investor, despite the fact that the latter must pay a greater bonus. The parameters used are $A=0.3, R=1, W_{0}=1, \sigma_{P}^{2}=\sigma_{Q}^{2}=1, b_{0}=0.2, \rho=0.3$, $\mu_{Q}=1.2$. 

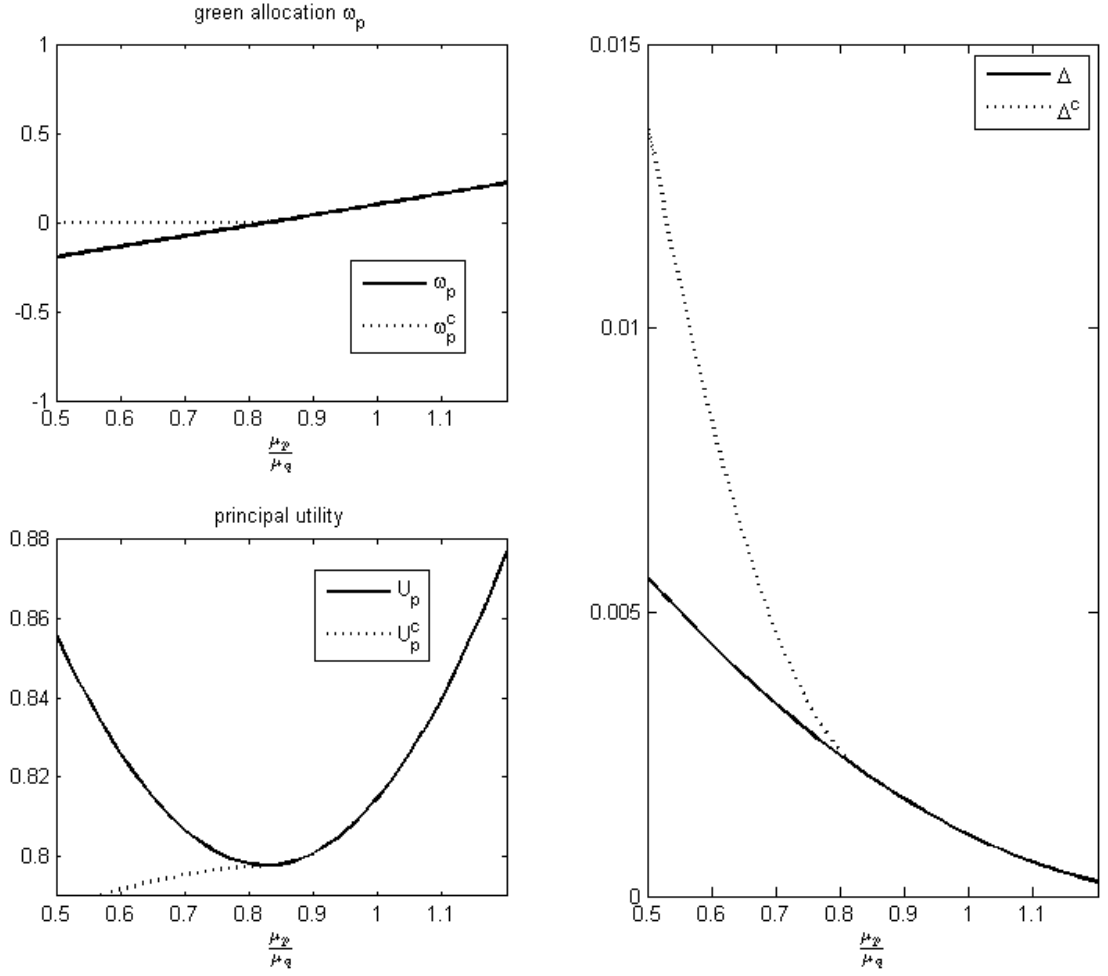

Figure 6: Effects of a short-selling constraint imposed only on the green contract. The allocation (top-left), the principal utility (bottom-left), and the optimal green bonus (righthand panel) are plotted against the ratio between $\mu_{P}$ and $\mu_{Q}$. When the constraint is binding the constrained optimal allocation $\omega_{p}^{c}$ is zero, the principal utility $U_{p}^{c}$ is lower than the utility without short-selling constraint $U_{p}$ and the green bonus $\bar{\Delta}^{c}$ is greater than the unconstrained one $\bar{\Delta}$. The parameters used are $A=0.3, R=1.11, W_{0}=1$, $\sigma_{P}^{2}=\sigma_{Q}^{2}=1, b_{0}=0.2, \rho=0.3, \mu_{Q}=1.2$. 

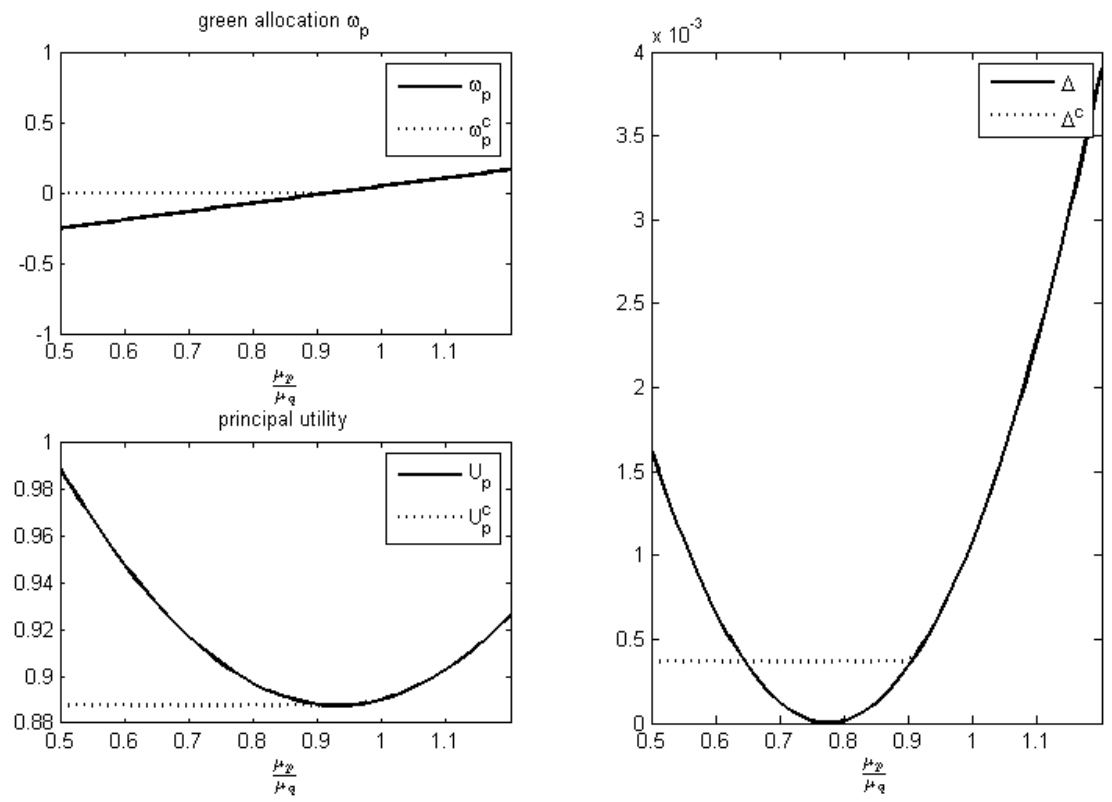

Figure 7: Effects of short-selling constraint imposed on the green and the conventional contract. The allocation (top left), the principal utility (bottom left), and the optimal green bonus (right-hand panel) are plotted against the ratio between $\mu_{P}$ and $\mu_{Q}$. When the constraint is binding the optimal allocation $\omega_{p}^{c}$ is zero, the principal utility $U_{P}^{c}$ is lower than the case without short-selling constraint $U_{p}$ and the green bonus $\bar{\Delta}^{c}$ can be greater or lower than the unconstrained $\bar{\Delta}$. The parameters used are $A=0.3, R=1, W_{0}=1$, $\sigma_{P}^{2}=\sigma_{Q}^{2}=1, b_{0}=0.2, \rho=-0.5, \mu_{Q}=1.2$. 